PROCEEDINGS OF THE

AMERICAN MATHEMATICAL SOCIETY

Volume 132, Number 8, Pages 2315-2319

S 0002-9939(04)07460-X

Article electronically published on February 19, 2004

\title{
DISCS IN COMPLEX MANIFOLDS WITH NO BOUNDED PLURISUBHARMONIC FUNCTIONS
}

\author{
JEAN-PIERRE ROSAY
}

(Communicated by Mei-Chi Shaw)

\begin{abstract}
Roughly speaking: In a complex manifold on which all bounded plurisubharmonic functions are constant, the center of a holomorphic disc and its boundary can be prescribed somewhat arbitrarily.
\end{abstract}

\section{INTRODUCTION}

Let $\bar{\Delta}$ be the closed unit disc in $\mathbb{C}$.

Theorem. Let $\mathcal{M}$ be a complex manifold, equipped with some Riemannian metric. The following are equivalent.

(1) Every bounded plurisubharmonic function on $\mathcal{M}$ is constant.

(2) For every $p \in \mathcal{M}$ and every nonempty open subset $\omega$ of $\mathcal{M}$, and for every $\varepsilon>0$, there exists a holomorphic map $\varphi$ from a neighborhood of $\bar{\Delta}$ in $\mathbb{C}$ into $\mathcal{M}$ such that $h(0)=p$ and $h\left(e^{i \theta}\right) \in \omega$ for all $\theta \in[0,2 \pi)-E_{\varepsilon}$, where $E_{\varepsilon}$ is a subset of $[0,2 \pi)$ of measure at most $\varepsilon$.

(3) For every $p \in \mathcal{M}$ and every continuous map $\lambda$ from the unit circle in $\mathbb{C}$ into $\mathcal{M}$, and for every $\varepsilon>0$, there exists a holomorphic map $h$ from a neighborhood of $\bar{\Delta}$ in $\mathbb{C}$ into $\mathcal{M}$ such that: $h(0)=p$ and $\operatorname{dist}\left(h\left(e^{i \theta}\right), \lambda\left(e^{i \theta}\right)\right) \leq \varepsilon$ for all $\theta \in[0,2 \pi)-E_{\varepsilon}$, where again $E_{\varepsilon}$ is a set of measure at most $\varepsilon$.

The above theorem seems close to being optimal. It cannot be hoped that the exceptional set $E_{\varepsilon}$ can be chosen to be an interval, as is however the case in the simple situation $\mathcal{M}=\mathbb{C}^{n}$.

The following proposition makes the point.

Proposition. Let $\mathcal{M}$ be a compact Riemann surface of genus $\geq 2$. Let $p$ and $q \in \mathcal{M}$, with $p \neq q$. Let $\delta>0$. There exists a neighborhood $\omega$ of $q$ such that if $h$ is a holomorphic map from a neighborhood of $\bar{\Delta}$ into $\mathcal{M}$ with $h(0)=p$ and if $I$ is an interval of length $>\delta$ on the unit circle, then $h(I)$ cannot be included in $\omega$.

So we could not even take as exceptional set $\left(h\left(e^{i \theta}\right) \notin \omega\right)$ a large interval of measure $2 \pi-\delta$.

In the theorem, $(3) \Rightarrow(2)$ is obvious (taking $\lambda$ constant). $(2) \Rightarrow(1)$ is immediate: Assume that (2) holds, and let $u$ be a bounded plurisubharmonic function on $\mathcal{M}$. Let $p$ and $q \in \mathcal{M}$. Take $\omega$ to be a small neighborhood of $q$. (2) gives a map $\varphi$ with

Received by the editors April 24, 2003.

2000 Mathematics Subject Classification. Primary 32H02, 32U05, 32Q65.

Partly supported by NSF. 
$h(0)=p$ and $h\left(e^{i \theta}\right) \in \omega$ except for a set of $\theta$ s of measure at most $\varepsilon$. The mean value property for subharmonic functions yields

$$
u(p) \leq \int_{0}^{2 \pi} u \circ h\left(e^{i \theta}\right) \frac{d \theta}{2 \pi} .
$$

Shrinking $\omega$ and letting $\varepsilon$ tends to 0 , one gets in the limit $u(p) \leq u(q)$. Similarly $u(q) \leq u(p)$; so $u$ is constant.

$(1) \Rightarrow(2)$ was first proved in full generality in [4] (corollary to the main theorem). See also [2], and see 1], 3] for earlier results. See [5] for a simpler and very different proof.

So it only remains to prove $(2) \Rightarrow(3)$.

Preliminary comments. There is no new ingredient in the proof of the theorem. We do not give a direct proof of $(1) \Rightarrow(3)$. We have to use (2) as a first step. Then we have to repeat a construction of holomorphic discs that is used in proving $(1) \Rightarrow$ (2). This is inspired from the fundamental work of Poletsky [3]. It is however worth pointing out the following. In proving $(1) \Rightarrow(2)$ the construction of discs comes only in the proof of a very general result on the plurisubharmonicity of functions defined by the infimum of averages over the boundary of holomorphic discs (theorem in [4). (2) is then obtained not directly but by a functional argument (not surprisingly since one has to finally use the hypothesis!). The argument is extremely simple but I have not been able to modify it in order to get a soft proof of $(2) \Rightarrow(3)$.

The proof will heavily rely on [5] (an alternate approach would rely on [4]).

\section{PROOF OF THE THEOREM}

II.1. As already said we have only to prove $(2) \Rightarrow(3)$. Let $\varepsilon>0$. We select disjoint closed intervals $I_{j} \subset[0,2 \pi), j=1, \ldots, J$, and $\theta_{j} \in I_{j}$ such that:

$$
\left|[0,2 \pi)-\bigcup_{j=1}^{I} I_{j}\right| \leq \frac{\varepsilon}{2}\left(|| \text { denotes measure), and if } \theta \in J_{j}, \operatorname{dist}\left[\lambda(\theta), \lambda\left(\theta_{j}\right)\right] \leq \frac{\varepsilon}{3} .\right.
$$

Using (2), for each $j \in\{1, \ldots, J\}$ we select a holomorphic map $g_{j}$ from a neighborhood of $\bar{\Delta}$ into $\mathcal{M}$ such that $g_{j}(0)=p$ and

$$
\operatorname{dist}\left(g_{j}\left(e^{i \nu}\right), \lambda\left(\theta_{j}\right)\right) \leq \frac{\varepsilon}{3}
$$

for all $\nu \in[0,2 \pi)=E_{j}$, where $E_{j}$ is a subset of $[0,2 \pi)$ of measure at most $\frac{\varepsilon}{2}$.

Next, we "put the $g_{j}$ 's together" and we claim that there is a $\mathcal{C}^{\infty}$ map $\Phi$ defined on a neighborhood of $\bar{\Delta} \times \bar{\Delta}$ in $\mathbb{C}_{z, w}^{2}$ into $\mathcal{M}$ such that:

$$
\left\{\begin{array}{l}
\Phi(z, 0) \equiv p . \\
\text { For every } z, w \mapsto \Phi(z, w) \text { is holomorphic in } w . \\
\text { If } \theta_{j} \in I_{j}, \text { then } \Phi\left(e^{i \theta_{j}}, w\right)=g_{j}(w) . \\
\frac{\partial \Phi}{\partial \bar{z}} \text { vanishes to infinite order along }|z|=1 .
\end{array}\right.
$$

The construction is fairly easy. For each $j \in\{1, \ldots, J\}$, let $S_{j}$ be the interval on the circle, $S_{j}=\left\{e^{i \theta}, \theta \in I_{j}\right\}$. Let $\chi_{j}(j=1, \ldots, J)$ be smooth functions on $\mathbb{C}$, with support respectively in small neighborhoods of $S_{j}$ with disjoint closures, such that $\chi_{j} \equiv 1$ on $S_{j}, 0 \leq \chi_{j}\left(e^{i \theta}\right) \leq 1$ and $\bar{\partial} \chi_{j}$ vanishes to infinite order along the unit circle. By additional cutoff we can impose $\left|\chi_{j}\right|<1+\varepsilon_{j}$ where $\varepsilon_{j}$ is chosen so that 
$g_{j}(\omega)$ is defined for $|w|<1+\varepsilon_{j}$. Off the unit circle, $\chi_{j}$ is not real valued. Then $\Phi$ can be defined in the following way:

$$
\left\{\begin{array}{l}
\text { For } z \text { near } S_{j} \text { (take disjoint neighborhoods), } \Phi(z, w)=g_{j}\left(\chi_{j}(z) w\right) . \\
\text { Away from } \bigcup S_{j}, \Phi(z, w)=p .
\end{array}\right.
$$

Note that by construction the set of $(\theta, \nu) \in[0,2 \pi)^{2}$ such that dist $\left[\Phi\left(e^{i \theta}, e^{i \nu}\right), \lambda(\theta)\right]$ $>\frac{2 \varepsilon}{3}$ has measure at most $2 \pi \varepsilon$. Indeed, it is included in the union of $\left\{(\theta, \nu), \theta \notin J_{j}\right\}$, which has measure at most $\frac{\varepsilon}{2} \times 2 \pi$, and of $\bigcup_{j}\left\{(\theta, \nu), \theta \in I_{j}, \nu \in E_{j}\right\}$, which has measure at most $\sum\left|I_{j}\right| \frac{\varepsilon}{2}$. Since the map $\left(e^{i \theta}, e^{i \nu}\right) \mapsto\left(e^{i \theta}, e^{i N \theta} e^{i \nu}\right)$ is area-preserving on the torus, for every integer $N$ the set

$$
\left\{(\theta, \nu) \in[0,2 \pi)^{2} ; \operatorname{dist} \Phi\left[\left(e^{i \theta}, e^{i N \theta} e^{i \nu}\right), \lambda(\theta)\right] \geq \frac{2 \varepsilon}{3}\right\}
$$

has measure at most $2 \pi \varepsilon$.

So there exists $\nu_{N}$ such that

$$
\begin{gathered}
\operatorname{dist}\left[\Phi\left(e^{i \theta}, e^{i N \theta} e^{i \nu_{N}}\right), \lambda(\theta)\right] \leq \frac{2 \varepsilon}{3} \\
\text { except for } \theta \text { in a set } E_{N} \subset[0,2 \pi), \text { with }\left|E_{N}\right| \leq \varepsilon .
\end{gathered}
$$

The above argument is of course borrowed from [3]. For $N \in \mathbb{N}$, set $\varphi_{N}(z)=$ $\Phi\left(z, e^{i \nu_{N}} z^{N}\right)$. Then the $\varphi_{N}$ are maps from neighborhoods of $\bar{\Delta}$ into $\mathcal{M}$ that satisfy:

$$
\left\{\begin{array}{l}
\varphi_{N}(0)=p \\
\operatorname{dist}\left(\varphi_{N}\left(e^{i \theta}, \lambda(\theta)\right)\right) \leq \frac{2 \varepsilon}{3} \text { except for } \theta \in E_{N} \\
\bar{\partial} \varphi_{N} \text { tends uniformly to } 0 \text { on } \bar{\Delta}
\end{array}\right.
$$

The last property follows immediately from $(*)$.

Note that there is no control of $\varphi_{N}$ off $\Delta$, and that there is no control on $\partial \varphi_{N}$.

Since $\bar{\partial} \varphi_{N}$ tends uniformly to 0 on $\bar{\Delta}$, we would like to conclude that there are holomorphic maps $h_{N}$ defined on neighborhoods of $\bar{\Delta}$ into $\mathcal{M}$ such that $h_{N}(0)=$ $\varphi_{N}(0)=p$ and

$$
\sup _{|z| \leq 1}\left[\operatorname{dist} \varphi_{N}(z), h_{N}(z)\right] \leq \frac{\varepsilon}{3}
$$

for $N$ large enough. We would then have $\operatorname{dist}\left(h_{N}\left(e^{i \theta}\right), \lambda(\theta)\right) \leq \varepsilon$ except for $\theta \in E_{N}$; so we could take $h=h_{N}$.

This is a question of uniformly approximating maps with small $\bar{\partial}$ by holomorphic maps. The question has been discussed in detail in [4]. Although it has a negative general answer, there are special cases where the answer is positive.

II.2. We now describe the result proved in 4, before finishing the proof of the theorem in II.3.

We are given in a complex manifold $\widetilde{\mathcal{M}}$ open subsets $\Omega_{0}, \ldots, \Omega_{R}$ with $K_{1}, \ldots, K_{R}$ compact subsets of $\Omega_{0}, \ldots, \Omega_{R}$ respectively. We assume that each $\Omega_{j}$ is biholomorphic to some open set in $\mathbb{C}^{k}(k=\operatorname{dim} \widetilde{\mathcal{M}})$. Finally, we are given open sets $U_{0}, \ldots, U_{R}$ in $\mathbb{C}$ such that $\bar{\Delta} \subset \bigcup_{j=0}^{R} U_{j}$ and with empty triple intersections, i.e., $U_{j} \cap U_{k} \cap U_{\ell}=\phi$ if $j k \ell$ are all distinct.

A map $\widetilde{\varphi}$ from $\Delta$ into $\widetilde{\mathcal{M}}$ is then called restricted (relative to the above data) if

$$
\widetilde{\varphi}\left(U_{j} \cap \Delta\right) \subset K_{j} \text { for all } j \in\{0, \ldots, R\} .
$$


We equip $\widetilde{\mathcal{M}}$ with some Riemannian metric. The main result of 4 is Proposition 1 in [4]:

For every $\varepsilon>0$ there exists $\delta>0$ such that if $u$ is a restricted map from $\Delta$ into $\widetilde{\mathcal{M}}$ satisfying $|\bar{\partial} u| \leq \delta$, there exists a holomorphic map $h$ from $\Delta$ into $\widetilde{\mathcal{M}}$ such that $h(0)=u(0)$ and

$$
\sup _{z \in \Delta}[\operatorname{dist}(u(z), h(z))]<\varepsilon .
$$

If $u$ is continuous on $\bar{\Delta}$, then we can find $h$ holomorphic on a neighborhood of $\bar{\Delta}$, simply replacing $h(z)$ by $h[(1-\eta) z]$ for $\eta>0$ small enough.

Note: In Proposition 1 in [4] it is absolutely crucial that no shrinking of the disc be allowed and that no uniform bound on $\partial u$ be imposed.

But, when considering only restricted maps, as above, there is an obvious bound of the kind:

$$
|\partial u(z)| \leq \frac{c}{1-|z|}
$$

Such a bound is not enough by itself in order to get a positive answer to the approximation problem for maps with small $\bar{\partial}$. Indeed, one can easily check that in Lempert's example described in [4] there is such a bound; however, approximation fails.

II.3. At the end of II.1 we had to approximate nonholomorphic maps $\varphi_{N}$ by holomorphic maps $h_{N}$. To put ourselves in the setting of II.2 we have to replace $\mathcal{M}$ by $\widetilde{\mathcal{M}}=\mathcal{M} \times \mathbb{C}$ and the map $\Phi$ by $\widetilde{\Phi}$ defined by $\widetilde{\Phi}(z, w)=(\Phi(z, w), w)$. The advantage is that for $j=1, \ldots, J$, the map $w \longrightarrow\left(g_{j}(w), w\right)=\widetilde{g}_{j}(w)$ is now an embedding of a neighborhood of $\bar{\Delta}$ with $\widetilde{\mathcal{M}}$. By a theorem of Royden [6] (see also [1], 4] Lemma 1.1), $\widetilde{g}_{j}(\bar{\Delta})$ has a neighborhood $\Omega_{j}$ biholomorphic to an open set in $\mathbb{C}^{k}$. For $j=1, \ldots, J, K_{j}$ will be a compact neighborhood of $\widetilde{g}_{j}(\bar{\Delta}) \subset \Omega_{j}$. Take $K_{0}=\{p\} \times \bar{\Delta}$ and $\Omega_{0}$ a small neighborhood of $K_{0}$ biholomorphic to some open set in $\mathbb{C}^{k}$.

Take $U_{0}$ open in $\mathbb{C}$ such that for $z \in U_{0} \cap \Delta, \Phi(z, w)=p$; so $\widetilde{\Phi}(z, w)=(p, w)$. For $j \in\{1, \ldots, J\}$, take $U_{j}$ open in $\mathbb{C}$ containing $I_{j}$, such that for $z \in U_{j} \cap \Delta$, $\widetilde{\Phi}(z, w) \in K_{j}$. To do this, in order to have $\widetilde{g}_{j}\left(\chi_{j}(z) \bar{\Delta}\right) \subset K_{j}$ for $j=1, \ldots, J$, we may have to replace $\chi_{j}$ by $\tau \circ \chi_{j}$ where $\tau$ is a smooth function on $\mathbb{C}$ such that $\tau(z)=z$ if $|z| \leq 1$ and $\sup |\tau| \leq 1+\eta$ for appropriate small $\eta$.

All the above can be done so that $\bar{\Delta} \subset \bigcup_{j=0}^{J} U_{j}$ and $U_{j} \cap U_{k}=\phi$ if $1 \leq j<k \leq J$. So there is no triple intersection.

For the map $\widetilde{\varphi}_{N}$ defined by

$$
\widetilde{\varphi}_{N}(z)=\left(\varphi_{N}\left(z, e^{i \nu_{N}} z^{N}\right), z\right)
$$

we are now in the situation described in II.2. So we can find holomorphic maps $\widetilde{h}_{N}$ from neighborhoods of $\bar{\Delta}$ into $\widetilde{\mathcal{M}}$ such that

$$
\sup _{|z| \leq 1} \operatorname{dist}\left[\widetilde{h}_{N}(z), \widetilde{\varphi}_{N}(z)\right] \underset{N \rightarrow \infty}{\longrightarrow} 0 .
$$

Finally, take $h_{N}=\pi \circ \widetilde{h}_{N}$ where $\pi$ is the natural projection of $\widetilde{\mathcal{M}}=\mathcal{M} \times \mathbb{C}$ onto $\mathcal{M}$. As explained at the end of II.1, this finishes the proof of the theorem. 


\section{ProOF OF THE PROPOSITION}

Let $\chi: \Delta \rightarrow \mathcal{M}$ be a holomorphic covering map ( $\Delta$ the open unit disc). We can assume $\chi(0)=p$. Let

$$
d=\min _{\chi(\zeta)=q}|\zeta| .
$$

Let $\delta>0$.

Claim: There exists $r>0$ such that if $g$ is a holomorphic map from $\Delta$ into $\Delta$ with $g(0)=0$ and $\left|\zeta_{0}\right| \geq d$, then $\left|g\left(e^{i \theta}\right)-\zeta_{0}\right| \leq r$ can hold for a set of $\theta s \in[0,2 \pi)$ of measure at most $\delta$.

This is a trivial consequence of the inequality

$$
\log \left|g(0)-\zeta_{0}\right| \leq \int_{0}^{2 \pi} \log \left|g\left(e^{i \theta}\right)-\zeta_{0}\right| \frac{d \theta}{2 \pi}
$$

Take $r$ as in the claim.

Then take a connected open neighborhood $\omega$ of $q$ so small that each connected component of its preimage under the map $\chi$ has diameter $\leq r$.

Finally, if $h$ is a holomorphic map from a neighborhood of $\bar{\Delta}$ into $\mathcal{M}$ with $h(0)=p$, lift this map to a holomorphic map $g(h=\chi \circ g)$ with $g(0)=0$.

If $I$ is an interval on the unit circle of length $>\delta$, then $h$ cannot map $I$ in $\omega$, since $g$ would map $I$ into one of the connected components of $\chi^{-1}(\omega)$. It would contradict the choice of $r$ (taking $\zeta_{0}$ in that component with $\chi\left(\zeta_{0}\right)=q$ ).

\section{REFERENCES}

[1] F. Lárusson and R. Sigurdsson, Plurisubharmonic functions and analytic discs on manifolds, J. Reine Angew. Math. 501 (1998), 1-39. MR 99e:32020

[2] A. Edigarian, A note on J. P. Rosay's paper: "Poletsky theory of disks on holomorphic manifolds", Ann. Polon. Math. 80 (2003), 125-132.

[3] E. Poletsky, Plurisubharmonic functions as solutions of variational problems, Proc. Sympos. Pure Math. 52 (1991), 163-171. MR 92h:32022

[4] J.-P. Rosay, Poletsky theory of disks on holomorphic manifolds, Indiana Univ. Math. J. 52 (2003), 157-169. MR 2004a:32053

[5] J.-P. Rosay, Approximation of non-holomorphic maps, and Poletsky theory of discs, J. Korean Math. Soc. 40 (2003), no. 3, 423-434. MR 2004c:32065

[6] H. L. Royden, The extension of regular holomorphic maps, Proc. Amer. Math. Soc. 43 (1974), 306-310. MR 49:629

Department of Mathematics, University of Wisconsin, Madison, Wisconsin 53706

E-mail address: jrosay@math.wisc.edu 